\title{
A Role for the Left Angular Gyrus in Episodic Simulation and Memory
}

\author{
DPreston P. Thakral, Kevin P. Madore, and Daniel L. Schacter \\ Department of Psychology, Harvard University, Boston, Massachusetts 02138
}

Functional magnetic resonance imaging (fMRI) studies indicate that episodic simulation (i.e., imagining specific future experiences) and episodic memory (i.e., remembering specific past experiences) are associated with enhanced activity in a common set of neural regions referred to as the core network. This network comprises the hippocampus, medial prefrontal cortex, and left angular gyrus, among other regions. Because fMRI data are correlational, it is unknown whether activity increases in core network regions are critical for episodic simulation and episodic memory. In the current study, we used MRI-guided transcranial magnetic stimulation (TMS) to assess whether temporary disruption of the left angular gyrus would impair both episodic simulation and memory (16 participants, 10 females). Relative to TMS to a control site (vertex), disruption of the left angular gyrus significantly reduced the number of internal (i.e., episodic) details produced during the simulation and memory tasks, with a concomitant increase in external detail production (i.e., semantic, repetitive, or off-topic information), reflected by a significant detail by TMS site interaction. Difficulty in the simulation and memory tasks also increased after TMS to the left angular gyrus relative to the vertex. In contrast, performance in a nonepisodic control task did not differ statistically as a function of TMS site (i.e., number of free associates produced or difficulty in performing the free associate task). Together, these results are the first to demonstrate that the left angular gyrus is critical for both episodic simulation and episodic memory.

Key words: angular gyrus; episodic memory; episodic simulation; fMRI; parietal cortex; TMS

\section{Significance Statement}

Humans have the ability to imagine future episodes (i.e., episodic simulation) and remember episodes from the past (i.e., episodic memory). A wealth of neuroimaging studies have revealed that these abilities are associated with enhanced activity in a core network of neural regions, including the hippocampus, medial prefrontal cortex, and left angular gyrus. However, neuroimaging data are correlational and do not tell us whether core regions support critical processes for simulation and memory. In the current study, we used transcranial magnetic stimulation and demonstrated that temporary disruption of the left angular gyrus leads to impairments in simulation and memory. The present study provides the first causal evidence to indicate that this region is critical for these fundamental abilities.

\section{Introduction}

Episodic simulation refers to the ability to imagine hypothetical episodes that might occur in the personal future (Schacter et al., 2008). According to the "constructive episodic simulation hypothesis" (Schacter and Addis, 2007), episodic simulation depends to a large extent on episodic memory. That is, episodic

Received May 15, 2017; revised July 14, 2017; accepted July 19, 2017.

Author contributions: P.P.T., K.P.M., and D.L.S. designed research; P.P.T. and K.P.M. performed research; P.P.T. and K.P.M. analyzed data; P.P.T., K.P.M., and D.L.S. wrote the paper.

This work was supported by the National Institute of Mental Health-National Institutes of Health (Grant R01MH60941 to D.L.S.). We thank Jyotika Bindra for assistance in data acquisition, Aleea Devitt for assistance in data analysis, and Scott Slotnick for TMS consultation.

The authors declare no competing financial interests.

Correspondence should be addressed to Preston P. Thakral, Department of Psychology, Harvard University, 33 Kirkland Street, Cambridge, MA 02138. E-mail: prestonthakral@fas.harvard.edu.

DOI:10.1523/JNEUROSCI.1319-17.2017

Copyright $\odot 2017$ the authors $\quad 0270-6474 / 17 / 378142-08 \$ 15.00 / 0$ memory supports both access to episodic details (e.g., who, what, and where information) and constructive processes that allow the recombination of those details into an imagined episode. Support for this hypothesis comes from several sources (for reviews, see Schacter et al., 2012, 2017b) including numerous functional magnetic resonance imaging ( $\mathrm{fMRI}$ ) studies demonstrating that both episodic simulation and memory elicit activity increases in a common core network of brain regions (for reviews and meta-analyses, see Benoit and Schacter, 2015; Stawarczyk and D'Argembeau, 2015). This network includes the hippocampus, medial prefrontal cortex, and left angular gyrus, among others. However, $\mathrm{AMRI}$ data are correlational and provide an indirect measure of neural activity. Therefore, an open question is whether core network regions make critical contributions to episodic simulation.

One answer to this question comes from neuropsychological research examining the consequences of hippocampal damage for simulation (for reviews, see Moscovitch et al., 2016; Schacter 
et al., 2017a). Many patients with hippocampal damage who exhibit impaired episodic memory also exhibit difficulties in simulating future events and novel scenes (Hassabis et al., 2007; Andelman et al., 2010; Race et al., 2011; Kurczek et al., 2015; but see, Squire et al., 2010; Dede et al., 2016). However, outside of the hippocampus, there is virtually no evidence that core network regions support critical processes for episodic simulation.

Relevant to the present study, there is evidence that the lateral parietal cortex contributes to episodic memory (for review, see Berryhill, 2012). For example, lesions encompassing the lateral parietal cortex impair the subjective experience associated with a variety of episodic memory judgments (Davidson et al., 2008; Berryhill et al., 2009; Drowos et al., 2010; Simons et al., 2010, Stamenova et al., 2017; Hower et al., 2014; Ciaramelli et al., 2017). Lateral parietal lesions also impair the ability to recall information (Berryhill et al., 2007; Ben-Zvi et al., 2015). Although studies of neuropsychological patients require interpretive caution that arises from the frequent occurrence of nonspecific lesions and associated symptomatology (for discussion, see Berryhill, 2012), transcranial magnetic stimulation (TMS) with non-brain-damaged individuals has been used to selectively target certain lateral parietal regions to assess their involvement in episodic memory. For example, inhibitory TMS to the left angular gyrus has been shown to reduce the accuracy of certain types of episodic memory judgments (Yazar et al., 2017), alter the bias of such judgments (Sestieri et al., 2013), and reduce their confidence (Yazar et al., 2014). Facilitatory TMS to this region enhances episodic memory performance (Wang et al., 2014; Nilakantan et al., 2017). With respect to episodic simulation, however, no study of healthy, non-brain-damaged people has provided direct evidence for a critical role of the lateral parietal cortex in episodic simulation of future events (but for a patient study using a related task, see Berryhill et al., 2010).

In the present study, we used inhibitory TMS to assess whether the lateral parietal cortex, specifically the left angular gyrus, is critically involved in episodic simulation as well as episodic memory. After TMS to the left angular gyrus or a control site (vertex), participants performed three tasks. In each task, participants were shown a cue word and asked either to imagine a future event (i.e., the episodic simulation task), recall a past event (i.e., the episodic memory task), or generate words related to the cue (i.e., the nonepisodic control task). We scored the simulation and memory responses using an adapted version of the Autobiographical Interview (Levine et al., 2002) to distinguish between "internal" and "external" details. Internal details are those that are episodic in nature (e.g., who, what, where, and when of the event) and external details are primarily semantic in nature. We tested the hypothesis that the left angular gyrus is critical for episodic processing by testing for a TMS site (left angular gyrus, vertex) by detail (internal, external) interaction (i.e., TMS should selectively impair the generation of internal/episodic details). To anticipate the results, TMS to the left angular gyrus relative to the vertex significantly reduced performance in the episodic tasks (i.e., participants generated fewer internal details during both simulation and memory) with no disruption in the control task. The current findings provide the first demonstration of a critical role of the left angular gyrus in episodic simulation and memory.

\section{Materials and Methods}

\section{General experimental design and statistical analysis}

The experimental design was approved by the Institutional Review Board of Harvard University. Written and informed consent was obtained from participants before the experiment commenced. All participants were native English speakers self-reported to be right handed and to have normal or corrected-to-normal vision. Participants were excluded if they might be pregnant, had a current or previous neuropsychiatric or neurological illness, were taking any psychoactive medications, had a prior head injury that required hospitalization, had a history of concussions, had experienced frequent or severe headaches, had a prior experience of a seizure, had a diagnosis or family history of epilepsy, or were diabetic. Before participating in the experiment, participants self-reported to not have consumed alcohol in the last $24 \mathrm{~h}$ and not to have consumed caffeinated drinks in the last $2 \mathrm{~h}$.

A within-participants design was used. Data from 17 participants were collected. One participant was excluded from the analysis due to excessive movement during the TMS session $(>5 \mathrm{~mm})$. The remaining 16 participants ( 10 females) had a mean age of 21.8 years (range $18-28)$. The final sample size of 16 was chosen to be identical with recent episodic memory TMS studies of the left lateral parietal cortex with similar within-participant designs (Sestieri et al., 2013; Wang et al., 2014; Nilakantan et al., 2017). Each participant received stimulation to the left angular gyrus and a control site (vertex). After application of TMS, participants completed three tasks: an episodic simulation task, an episodic memory task, and a nonepisodic control task (i.e., a free associate task). The order of the TMS site and of the three tasks were counterbalanced across participants. Repeated-measures ANOVAs with follow-up paired $t$ tests were conducted to assess TMS-induced effects. As noted in the Introduction, our primary interest was in the TMS site (left angular gyrus, vertex) by detail (internal, external) interaction. This analysis approach is identical to prior studies investigating the consequences of reduced episodic processing on internal/episodic and external/nonepisodic detail generation in the Autobiographical Interview in different experimental groups (e.g., in older vs young adults or patients vs controls; Levine et al., 2002; Berryhil et al., 2007; Addis et al., 2008, 2010; Gaesser et al., 2011; Madore et al., 2014; Devitt et al., 2017). All other ANOVA results are secondary and supplementary to this main analysis of interest and are reported for completeness. Dependent variables of interest are discussed in detail below (see "Variables of interest" section).

\section{Stimuli and tasks}

Stimuli comprised 30 words drawn from the Medical Research Council Psycholinguistics database (Coltheart, 1981; http://websites.psychology. uwa.edu.au/school/MRCDatabase/uwa_mrc.htm). Words selected were nouns with similar length (i.e., 3-4 letters) and high in familiarity, concreteness, and imagability (i.e., 540-700). The 30 word list was split into six separate sublists that did not vary with respect to average word length or the noted qualities. These six lists were counterbalanced across participants such that each word was used for each task and TMS site (i.e., three tasks and two TMS sites).

Each participant completed the simulation, memory, and free associate tasks. Tasks were completed in blocks of five trials. Before each task block, a task cue was presented for $1 \mathrm{~s}$ (Fig. 1). For each trial, participants were instructed to recite the cue word verbally first and then begin performing the given task. Each cue word was shown for $30 \mathrm{~s}$. After the $30 \mathrm{~s}$ period, a difficulty prompt appeared for $2 \mathrm{~s}$ and participants were instructed to rate how difficult it was to perform the respective task on a scale of 1 (not at all difficult) to 5 (extremely difficult). Participants responded with a keyboard using their left hand. The current experimental design was chosen to be similar to previous behavioral and neuroimaging studies using word-cued simulation, memory, and nonepisodic tasks (e.g., Addis et al., 2007, 2009, 2011; Madore and Schacter, 2016). Participants practiced the three tasks before application of TMS to the first site. There were no experimenter inputs or questions during the tasks to minimize methodological bias.

For the episodic simulation and memory tasks, participants were instructed to imagine or remember an event within the upcoming or past 5 years that somehow incorporated the cue. Participants were told that the cue words were meant to only serve as a trigger to help them imagine or remember an event. If participants could not imagine or remember an event using the cue word, they were instructed to use the cue as a springboard for another event that the cue reminded them of. The future or past event was to be outside of their routine and be specific in both time 
(i.e., last between only a few minutes to a few hours) and place. For example, if they imagined or remembered going on a vacation, participants were instructed to think about one event that happened to them on one day of the vacation (e.g., going to a beach for the afternoon) rather than the vacation as a whole (e.g., a week-long trip). They were instructed to think about the event from a first-person perspective and not as an outside observer looking in on themselves from an external vantage point. Participants were told to try to think of everything they could for the details of the event including the people involved, actions, and emotions (i.e., all possible details that contributed to the event in a realistic way). Participants were asked to describe the event verbally and to try and use the entire $30 \mathrm{~s}$ period to complete the tasks.

For the free associate task, participants were instructed to freely generate as many words that were semantically (or thematically) associated with the cue as they could. They were instructed to not think about the associated words in relation to themselves or their own life, but rather to generate associated words as if they were looking them up in a dictionary (i.e., to focus on words that factually make sense given the cue). As with the memory and simulation tasks, participants were told to use the entire $30 \mathrm{~s}$ period to generate as many words as possible.

It is important to discuss why the free associate task serves as an appropriate control task in the present experiment. First, the free associate task is similar to the memory and simulation task in that it requires the generation of semantic information but without the requirement to generate a coherent episodic event. Second, the free associate task is similar to other nonepisodic control tasks that have been used in fMRI studies identifying core network activity during episodic simulation and memory (for review, see Benoit and Schacter, 2015). Third, free associate production has been found to engage the left angular gyrus (e.g., Wende et al., 2012; Bonnici et al., 2016) and there is evidence to indicate that disruption of the left angular gyrus impairs performance in certain semantic memory tasks (e.g., Davey et al., 2015; Price et al., 2016). Together, the current free associate task can be considered a relatively strict control for nonepisodic processing (i.e., semantic retrieval).

\section{TMS procedure}

The left angular gyrus site (MNI coordinate: $-48,-64,30)$ was based on a previously reported meta-analysis of episodic simulation and memory fMRI studies (Benoit and Schacter, 2015). This coordinate corresponds to the peak voxel within a left angular gyrus cluster associated with the joint activation for episodic simulation and episodic memory (Fig. 2). Although the meta-analysis identified activity in the homologous area in the right hemisphere, we targeted the left hemisphere given prior neuroimaging evidence that shows predominantly left lateralized parietal cortex activity during episodic/autobiographical memory (Kim, 2010, 2012; Shimamura, 2011; Rugg and Vilberg, 2013; Bellana et al., 2016), as well as prior TMS studies of episodic memory to focus on the left lateral parietal cortex (Sestieri et al., 2013; Wang et al., 2014; Yazar et al., 2014, 2017; Nilakantan et al., 2017). The control site (vertex) was identified on each individual participant's anatomic image as the location at which the central sulci in each hemisphere met (for other TMS studies using a similar control condition, see Yazar et al., 2014, 2017; Blumenfeld et al., 2014; Ryals et al., 2016; Nilakantan et al., 2017).

The Brainsight (Rogue Research) neuronavigation system was used to target the left angular gyrus and vertex. Three anatomical land- marks were identified in external space (using a pointer), identified in Brainsight on the participants' anatomic image, and then coregistered. Reflective markers were attached to the TMS coil as well as the participant and emitted signals picked up by an infrared camera. This procedure allowed tracking of the TMS coil in real-time relative to the head (and linked anatomic image). A MagPro X100 Magnetic Stimulator equipped with a Cool-B65 A/P $75 \mathrm{~mm}$ coil was used to apply $1 \mathrm{~Hz}$ repetitive TMS at $70 \%$ of maximum output for $10 \mathrm{~min}$. This TMS protocol constitutes a standard inhibitory protocol (for other uses of this protocol see, Kosslyn, et al., 1999; Slotnick and Thakral, 2011; Thakral and Slotnick, 2011; see also, Chen et al., 1997). The coil was positioned approximately perpendicular to the stimulation site and maintained at an angle $45^{\circ}$ away from the midline (i.e., the TMS cable was oriented downward in the posterior direction). During the application of TMS, the coil was kept to a minimum of 1-2 mm away from the target location (i.e., left angular gyrus or vertex). During the $10 \mathrm{~min}$ of stimulation, participants made odd/even judgments to random numbers presented in the center of the screen. The three tasks were completed within $\sim 8 \mathrm{~min}$ of TMS offset. There was a $30 \mathrm{~min}$ rest period after TMS to each site to ensure that TMS effects had dissipated completely (Kosslyn et al., 1999; Slotnick and Thakral, 2011; Thakral and Slotnick, 2011).

\section{Variables of interest}

All responses were audio-recorded, transcribed, and coded for internal details, external details, and free associates. For the simulation and memory tasks, internal details were episodic in nature (e.g., people, actions, objects, thoughts, feelings and surroundings that focused on a central event of interest). External details were semantic in nature, repetitive, or off topic. The internal and external coding procedures were adapted from the original Autobiographical Interview developed by Levine et al. (2002) to segment individuals' descriptions of experiences into episodic and 

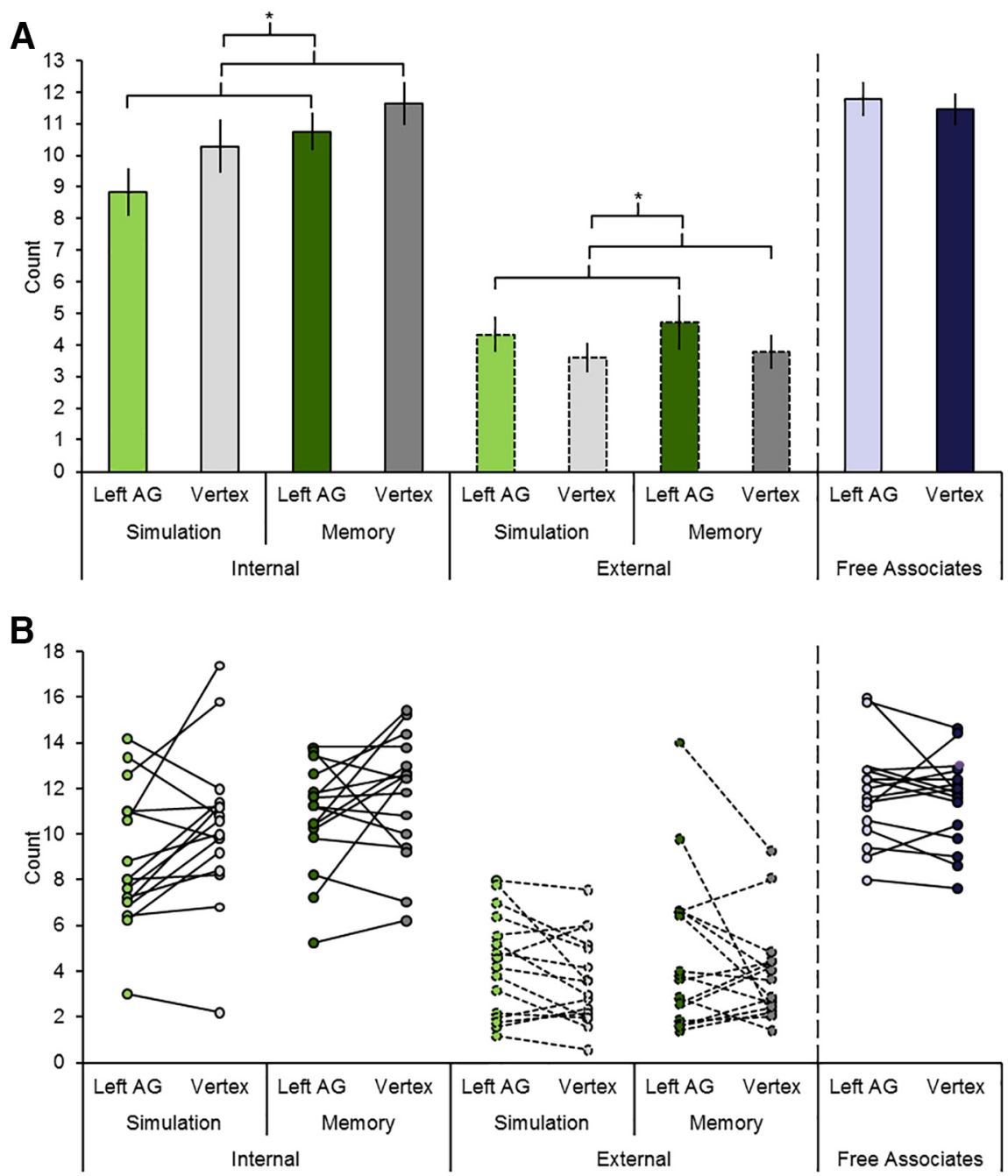

Figure 3. TMS results. A, Mean number of internal and external details for the simulation and memory tasks and the number of free associates produced with left angular gyrus TMS or vertex TMS. Error bars indicate mean \pm SEM. Asterisks indicate significant results (see main text for details). $\boldsymbol{B}$, Individual participant number of internal and external details for the simulation and memory tasks and number of free associates produced with left angular gyrus TMS or vertex TMS.

nonepisodic information (for examples of this coding scheme, see Addis et al., 2008; Gaesser et al., 2011; Madore et al., 2014; Madore and Schacter, 2016). All coding was conducted by a single rater who was blind to the TMS session. We confirmed interrater reliability with a separate rater who scored a random selection of 60 responses (with an equal number sampled from each task and TMS session). Interrater reliability was high, with a Cronbach's $\alpha$ of 0.95 for total details ( 0.94 for internal details and 0.82 for external details; these reliability measures are consistent with previous studies; e.g., Gaesser et al., 2011; Dede et al., 2016). For the free associate task, the number of responses generated served as the dependent variable (excluding repetitions). In addition, we investigated whether TMS to left angular gyrus relative to vertex modulated the difficulty with which participants experienced each task.

\section{Results}

Figure $3 A$ illustrates the mean number of internal and external details generated as a function of task (simulation and memory) and TMS site (left angular gyrus and vertex; see first eight bars). To assess the effect of TMS to the left angular gyrus during episodic simulation and memory on detail generation, we conducted a three-way ANOVA with factors TMS site (left angular gyrus, vertex), task (simulation, memory), and detail (internal, external). Although we computed the main effects and interac- tions for the different factors, we focus here on the interaction of TMS site by detail because it trumps the main effects and addresses our hypothesis most directly (see Introduction). The TMS site by detail interaction was found to be significant $\left(F_{(1,15)}=6.54, p=0.022\right.$, partial $\eta^{2}=$ $0.30)$. As predicted, follow-up paired $t$ tests revealed that TMS to the left angular gyrus relative to TMS to the vertex significantly reduced the number of internal details $\left(t_{(15)}=2.36, p=0.032, \mathrm{~d}=0.59\right.$; Fig. $3 \mathrm{~A}$, compare bars 1 and 3 vs 2 and 4; collapsed across the simulation and memory tasks, 14 of 16 participants demonstrated this effect; see Fig. $3 B$ for individual participant data split by the two tasks) and increased the number of external details $\left(t_{(15)}=\right.$ $2.29, p=0.037, \mathrm{~d}=0.57$; Fig. $3 A$, compare bars 5 and 7 vs 6 and 8; collapsed across the simulation and memory tasks, 10 of 16 participants demonstrated this effect; see Fig. $3 B$ for individual participant data split by the two tasks). These findings indicate that TMS to the left angular gyrus selectively impaired the generation of internal/episodic details.

The following ANOVA results are secondary and supplementary to the main analysis of interest (see above) and are reported for completeness. Replicating prior studies (e.g., Addis et al., 2010; Madore and Schacter, 2016), the ANOVA also revealed a main effect of task (i.e., the memory task was associated with more details than the simulation task $\left(F_{(1,15)}=\right.$ $11.14, p=0.004$, partial $\left.\eta^{2}=0.43\right)$ and a main effect of detail (i.e., greater internal relative to external details generated; $F_{(1,15)}=116.23, p=1.84 \times 10^{-8}$, partial $\left.\eta^{2}=0.89\right)$. The ANOVA failed to reveal a main effect of site $\left(F_{(1,15)}=0.81, p=\right.$ $0.38)$, a task by TMS site interaction $\left(F_{(1,15)}=1.14, p=0.30\right)$, a task by detail interaction $\left(F_{(1,15)}=0.77, p=0.40\right)$, and no threeway interaction $\left(F_{(1,15)}=0.06, p=0.81\right)$. The lack of a main effect of TMS site suggests that overall performance (i.e., internal and external detail production) was statistically equivalent after TMS to the left angular gyrus and vertex. The lack of a task by TMS site interaction suggests that the TMS-induced effects were of similar magnitude as a function of the simulation and memory tasks.

The crossover interaction as a function of TMS site and detail raises the question of whether TMS to the left angular gyrus reduced episodic processing (i.e., reduced the generation of internal details) or enhanced the processing associated with external detail production. First, we note that the TMS protocol used was inhibitory and assumed a priori to impair performance (Kosslyn et al., 1999; Slotnick and Thakral, 2011; Thakral and Slotnick, 2011). Therefore, if the left angular gyrus is associated with the processing of "external details," then TMS should have reduced the production of such details, but the opposite was observed. Nevertheless, to further elucidate the nature of the TMS-induced deficit, we examined performance on our nonepisodic control 

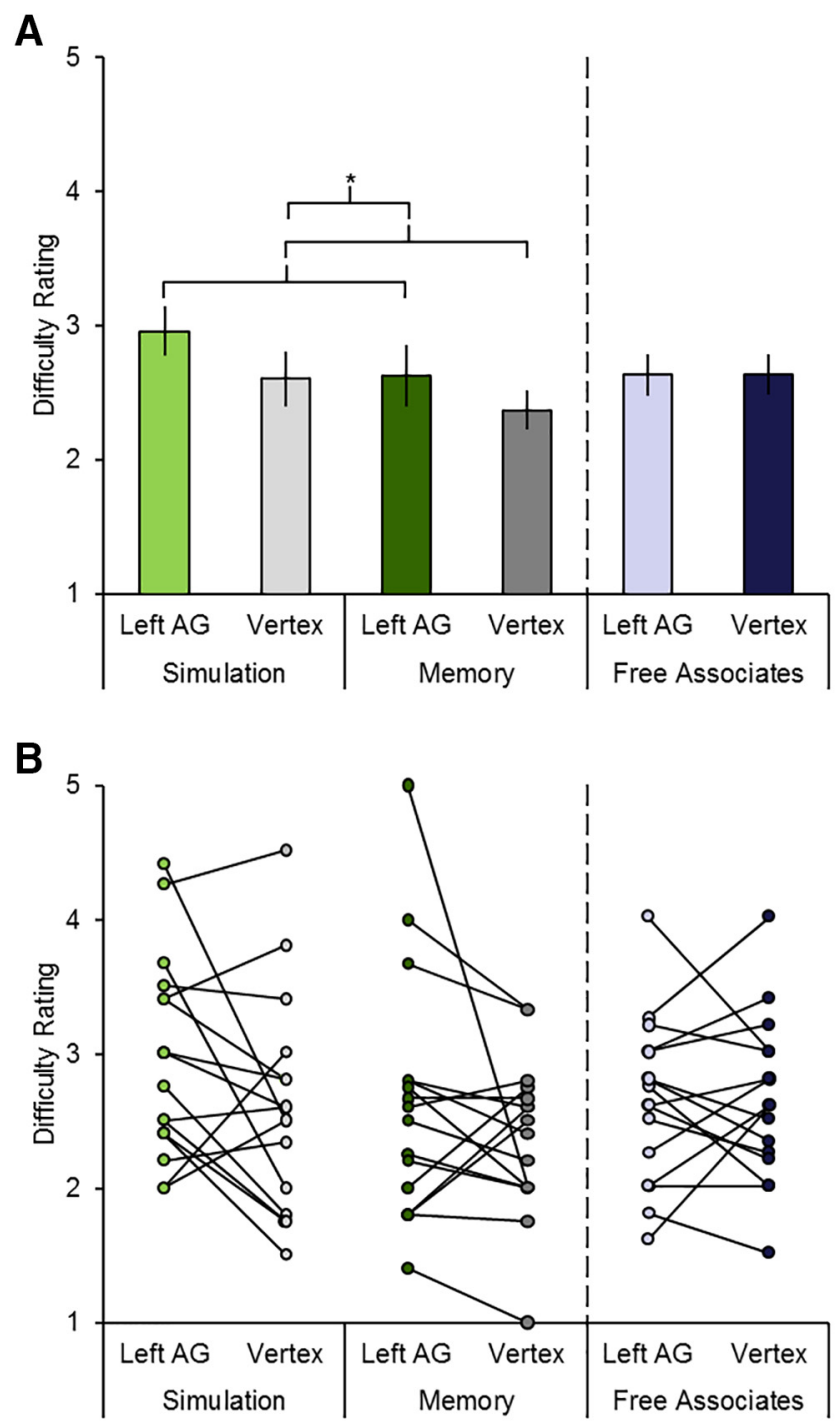

Figure 4. TMS results. $A$, Mean difficulty rating for each of the three tasks with left angular gyrus TMS or vertex TMS. Error bars indicate mean \pm SEM. Asterisks indicate significant results (see main text for details). $\boldsymbol{B}$, Individual participant difficulty ratings for each of the three tasks with left angular gyrus TMS or vertex TMS.

task, the free associate task. Free associate production did not differ as a function of TMS site $\left(t_{(15)}=0.81, p=0.43\right.$, paired $t$ test; Fig. $3 A$, compare bar 9 vs 10 ; see Fig. $3 B$ for individual participant data). In a further test of whether specifically episodic processing was impaired, we conducted a follow-up two-way ANOVA with factors TMS site (left angular gyrus, vertex) and task (simulation, memory, and free associate task) using the number of internal details as the dependent variable for the simulation and memory tasks (Fig. 3A, bars 1 through 4 and bars 9 and 10). This ANOVA revealed a significant task by TMS site interaction $\left(F_{(2,30)}=3.50, p=0.043\right.$, partial $\left.\eta^{2}=0.19\right)$. These results indicate that TMS to the left angular gyrus caused a specific deficit in episodic processing. The concomitant boost observed in external detail production is therefore a likely byproduct of deficient left angular gyrus-mediated episodic processing (for discussion of the relation between internal and external details in previous studies of episodic simulation and memory that is consistent with this interpretation, see Devitt et al., 2017).

Figure $4 A$ illustrates the mean difficulty rating associated with the three tasks. We first conducted an ANOVA restricted to the two episodic tasks with factors TMS site (left angular gyrus, vertex) and task (simulation, memory). This ANOVA revealed a significant main effect of site (i.e., greater difficulty after TMS to the left angular gyrus relative to the vertex; $F_{(1,15)}=5.17, p=$ 0.038 , partial $\eta^{2}=0.26$; Fig. $4 A$, compare bars 1 and 3 vs 2 and 4; collapsed across the simulation and memory tasks, 13 of 16 participants demonstrated this effect; see Fig. $4 B$ for individual participant data split by the two tasks). The main effect of task and the task by TMS site interaction was not significant $\left(F_{(1,15)}=\right.$ $2.72, p=0.12$, and $F_{(1,15)}=0.11, p=0.75$, respectively). As with the detail analysis above, the nonsignificant interaction suggests that the increase in difficulty after TMS to the left angular gyrus was equivalent in magnitude across the episodic tasks. The increase in difficulty was confirmed to be specific to the episodic tasks by a follow-up ANOVA with factors TMS site (left angular gyrus, vertex) and task (episodic, nonepisodic (i.e., free associate task)). This analysis revealed a significant task by TMS site interaction $\left(F_{(1,15)}=5.99, p=0.027\right.$, partial $\left.\eta^{2}=0.29\right)$, with no difference in difficulty as a function of TMS site for the free associate task $\left(t_{(15)}=0.02, p=0.99\right.$; Fig. $4 A$, compare bar 5 vs 6 ; see Fig. $4 B$ for individual participant data).

\section{Discussion}

The current study provides the first causal evidence to indicate that the left angular gyrus plays a critical role in episodic simulation and memory. After TMS to the left angular gyrus, participants generated fewer episodic/internal details for both future and past events and generated more nonepisodic/external details for each class of event. This effect was specific to episodic processing because TMS did not influence performance in the nonepisodic control task. We highlight that the finding of a crossover interaction as a function of TMS site and detail production (i.e., internal and external) is conceptually similar to previous findings from related tasks. For example, older adults, relative to young adults, demonstrate a similar trade-off between internal and external details when generating past and future events (i.e., older adults generate fewer internal and more external details relative to young adults; e.g., Levine et al., 2002; Addis et al., 2008, 2010; Gaesser et al., 2011; Madore et al., 2014). A number of experiments have supported the interpretation that these trade-offs likely reflect an age-related reduction in specifically episodic processing (e.g., Addis et al., 2008, 2010; Madore et al., 2014). In the present study, we demonstrated that TMS to the left angular gyrus relative to the vertex did not disrupt semantic retrieval in a free associate task. Together, our findings suggest that the increase in external detail production is compensation for the TMS-induced deficit in episodic processing mediated by the left angular gyrus (for related evidence from non-TMS studies that supports this interpretation, see Devitt et al., 2017).

An alternative to the above compensation account is that TMS to the left angular gyrus may have altered participants' retrieval orientation during the simulation and memory tasks. Retrieval orientation refers to a goal-directed processing strategy that individuals invoke when presented with a retrieval cue (Rugg and Wilding, 2000; Morcom and Rugg, 2012). In the current study, it is possible that, after disruption of episodic processing within the left angular gyrus, participants may have shifted to a more nonepisodic retrieval orientation, leading to a greater production of external relative to internal details. This interpretation is consistent with our finding that TMS to the left angular gyrus also led to a subjective experience of greater difficulty. Specifically, the reduced subjective experience of retrieved episodic details (in terms of difficulty or other factors not measured in the current study 
such as vividness or confidence) may have shifted participants toward the report of external relative to internal details. Regardless of whether the crossover interaction of TMS site and detail reflects compensation or a shift in retrieval orientation, the current study provides the first causal evidence to indicate that the left angular gyrus plays a critical role during simulation and memory.

Given the causal evidence provided by the current study, it is important to consider what precise function the left angular gyrus supports during episodic simulation and memory. A number of proposals have been put forth regarding the role of the lateral parietal cortex, specifically the left angular gyrus, during episodic memory (for reviews, see Levy, 2012; Berryhill, 2012; Rugg and Vilberg, 2013; Sestieri et al., 2017). For example, it has been proposed that the angular gyrus supports bottom-up attention to recollected content (Cabeza et al., 2012), serves to represent recollected content in an "episodic buffer" (Vilberg and Rugg, 2008), or binds episodic features into an integrated representation (Shimamura, 2011). Most directly relevant to the present findings, according to Guerin et al. (2012), the angular gyrus supports the retrieval of detailed information from episodic memory. The current findings are consistent with this latter proposal in that TMS to the left angular gyrus reduced the retrieval of episodic details for both past and future events. As noted above, because TMS to the left angular gyrus also led to an experience of greater difficulty, the present results are also consistent with proposals that highlight the role of the lateral parietal cortex in mediating the subjective experience during episodic memory (for review, see Moscovitch et al., 2016).

Despite the frequency with which neuroimaging studies have associated the left lateral parietal cortex with both episodic simulation and memory (for review, see Benoit and Schacter, 2015; see also, Vilberg and Rugg, 2008; Kim, 2010), the question remains as to why lateral parietal lesions tend to leave the accuracy of episodic memory intact while impairing more subjective aspects of both episodic memory (for reviews, see Berryhill, 2012; Moscovitch et al., 2016) and, as currently shown, episodic simulation. It is possible that lateral parietal activity seen with fMRI is epiphenomenal and/or that laboratory measures of episodic memory (e.g., tests of source memory) may simply be insensitive to parietal lesions (Simons et al., 2010; Sestieri et al., 2017). Relevant to this point, Shimamura (2011) proposed that it is the amount of binding during episodic retrieval that determines the engagement of the lateral parietal cortex (i.e., the more features of a given memory, the greater lateral parietal involvement). With respect to the paradigm used here, participants were required to construct a coherent past and future episode with relatively complex episodic information (e.g., people, place, and/or object information). In contrast, laboratory measures of episodic memory require the retrieval of only a single episodic feature (e.g., the spatial location of a previously presented item), which therefore requires less binding (Shimamura, 2011). Although more research is needed to further elucidate the role of the lateral parietal cortex in simulation and memory, the current and previous findings suggest that one role of the lateral parietal cortex is to support the retrieval of episodic details that comprise a complex event.

An important avenue for future research is to examine the role of the left angular gyrus in other cognitive domains. The left angular gyrus is a neural region that has been associated with processes other than episodic simulation and memory (for review, see Humphreys and Lambon Ralph, 2015). It is possible that the type of process recruited during episodic simulation and memory may also be recruited during other processes (e.g., Bon- nici et al., 2016; Sestieri et al., 2017). For example, a recent study applied transcranial direct current stimulation to the left angular gyrus and demonstrated that this region supports the integration of complex semantic information (Price et al., 2016). Related to this finding, Binder and Desai (2011) have argued that the left angular gyrus supports the representation of "event concepts" (for discussion, see Levy, 2012). An event concept contains both the information that defines a particular event (e.g., the cake and candles of a birthday party) and the series of events across space and time that define the event (e.g., blowing out candles followed by opening presents). It is possible that episodic simulation and memory are jointly supported by the angular gyrus through the representation of such event concepts. This proposal explains why TMS did not impair performance on the free associate task, because it did not necessitate the generation of an event, but did impair performance on tasks requiring the generation of a coherent event (i.e., the simulation and memory tasks). We note, though, that our effects were specific to episodic processing (i.e., internal detail generation), which is difficult to reconcile with purely semantic accounts of angular gyrus function (e.g., Binder and Desai, 2011).

There are some potential limitations of the current study. First, performance on the Autobiographical Interview can be driven by a variety of nonepisodic processes, such as narrative or descriptive style, which can affect the relative proportions of internal and external details that people produce during the Autobiographical Interview (Gaesser et al., 2011; Madore et al., 2014). Therefore, it is conceivable that TMS to the angular gyrus affected the narrative or descriptive style of participants (i.e., how they talk about events) rather than episodic retrieval per se. However, the evidence to date indicates that such considerations are most relevant to between-participant comparisons, such as younger versus older adults who differ in narrative style (Gaesser et al., 2011), as opposed to the within-participants approach taken here. Moreover, if TMS affected a broad process such as narrative or descriptive style, then we should have observed an acrossdetail deficit in performance as a function of TMS site. However, we did not observe this pattern (i.e., the significant TMS site by detail interaction).

Second, whereas our effects were specific to the left angular gyrus relative to the vertex, it is possible that the current results may have been due to TMS-induced effects in other core network regions such as the hippocampus. Related to this point, recent TMS studies have targeted specific lateral parietal regions that are functionally connected to the hippocampus and demonstrated that TMS to such regions can affect episodic memory performance (Wang et al., 2014; Nilakantan et al., 2017). These findings indicate that episodic memory is supported by interactions between the lateral parietal cortex and hippocampus. It will be important for future studies to use such methodologies to further elucidate the nature of regional interactions within the core network during episodic simulation.

Lastly, although we used individual participant anatomic data to target the TMS, the left angular gyrus location targeted for TMS was derived from a meta-analysis of episodic simulation and memory studies (Benoit and Schacter, 2015). Therefore, it is possible that the exact location within the angular gyrus associated with episodic processing may have varied across participants (cf., Mueller et al., 2013). Because significant effects were nonetheless observed, this potential problem was evidently not a major influence on the results. Regardless, this point highlights the need for experiments that use fMRI-guided TMS on an individual participant basis to increase anatomical specificity (e.g., Slot- 
nick and Thakral, 2011; Thakral and Slotnick, 2011; Wang et al., 2014; Nilakantan et al., 2017).

To conclude, the present findings clearly demonstrate the critical role of the left angular gyrus in episodic simulation and episodic memory. Together with prior evidence concerning the hippocampus (for review, see Schacter et al., 2017a), the present data provide support for the constructive episodic simulation hypothesis (Schacter and Addis, 2007), in that certain core network regions support common and critical processes during both episodic simulation and memory. Additional studies will be needed to shed light on more finegrained questions regarding the role of the left angular gyrus during episodic simulation and memory. For example, it is uncertain whether the left angular gyrus is critical for the initial construction of an episode or for subsequent processes that occur after initial construction (e.g., post-memory/simulation decision processes; for discussion, see Thakral et al., 2017). The results of the current study provide an important first step toward answering these and related questions regarding the neural basis of episodic simulation and memory.

\section{References}

Addis DR, Wong AT, Schacter DL (2007) Remembering the past and imagining the future: Common and distinct neural substrates during event construction and elaboration. Neuropsychologia 45:1363-1377. CrossRef Medline

Addis DR, Wong AT, Schacter DL (2008) Age-related changes in the episodic simulation of future events. Psychol Sci 19:33-41. CrossRef Medline

Addis DR, Pan L, Vu MA, Laiser N, Schacter DL (2009) Constructive episodic simulation of the future and the past: Distinct subsystems of a core brain network mediate imagining and remembering. Neuropsychologia 47:2222-2238. CrossRef Medline

Addis DR, Musicaro R, Pan L, Schacter DL (2010) Episodic simulation of past and future events in older adults: evidence from an experimental recombination task. Psychol Aging 25:369-376. CrossRef Medline

Addis DR, Cheng T, Roberts RP, Schacter DL (2011) Hippocampal contributions to the episodic simulation of specific and general future events. Hippocampus 21:1045-1052. CrossRef Medline

Andelman F, Hoofien D, Goldberg I, Aizenstein O, Neufeld MY (2010) Bilateral hippocampal lesion and a selective impairment of the ability for mental time travel. Neurocase 16:426-435. CrossRef Medline

Bellana B, Liu Z, Anderson JA, Moscovitch M, Grady CL (2016) Laterality effects in functional connectivity of the angular gyrus during rest and episodic retrieval. Neuropsychologia 80:24-34. CrossRef Medline

Benoit RG, Schacter DL (2015) Specifying the core network supporting episodic simulation and episodic memory by activation likelihood estimation. Neuropsychologia 75:450-457. CrossRef Medline

Ben-Zvi S, Soroker N, Levy DA (2015) Parietal lesion effects on cued recall following pair associate learning. Neuropsychologia 73:176-194. CrossRef Medline

Berryhill ME, Phuong L, Picasso L, Cabeza R, Olson IR (2007) Parietal lobe and episodic memory: bilateral damage causes impaired free recall of autobiographical memory. J Neurosci 27:14415-14423. CrossRef Medline

Berryhill ME, Drowos DB, Olson IR (2009) Bilateral parietal cortex damage does not impair associative memory for paired stimuli. Cogn Neuropsychol 26:606-619. CrossRef Medline

Berryhill ME, Picasso L, Arnold R, Drowos D, Olson IR (2010) Similarities and differences between parietal and frontal patients in autobiographical and constructed experience tasks. Neuropsychologia 48:1385-1393. CrossRef Medline

Berryhill ME (2012) Insights from neuropsychology: pinpointing the role of the posterior parietal cortex in episodic and working memory. Font Integr Neurosci 6:1-12. CrossRef Medline

Binder JR, Desai RH (2011) The neurobiology of semantic memory. Trends Cogn Sci 15:527-536. CrossRef Medline

Blumenfeld RS, Lee TG, D’Esposito M (2014) The effects of lateral prefrontal transcranial magnetic stimulation on item memory encoding. Neuropsychologia 53:197-202. CrossRef Medline

Bonnici HM, Richter FR, Yazar Y, Simons JS (2016) Multimodal feature integration in the angular gyrus during episodic and semantic retrieval. J Neurosci 36:5462-5471. CrossRef Medline

Cabeza R, Ciaramelli E, Moscovitch M (2012) Cognitive contributions of the ventral parietal cortex: An integrative theoretical account. Trends Cogn Sci 16:338-352. CrossRef Medline

Chen R, Classen J, Gerloff C, Celnik P, Wassermann EM, Hallett M, Cohen LG (1997) Depression of motor cortex excitability by low-frequency transcranial magnetic stimulation. Neurology 48:1398-1403. CrossRef Medline

Ciaramelli E, Faggi G, Scarpazza C, Mattioli F, Spaniol J, Ghetti S, Moscovitch M (2017) Subjective recollection independent from multifeatural context retrieval following damage to the posterior parietal cortex. Cortex 91:114-125. CrossRef Medline

Coltheart M (1981) The MRC psycholinguistic database. Q J Exp Psychol Sect A 33:497-505. CrossRef

Davey J, Cornelissen PL, Thompson HE, Sonkusare S, Hallam G, Smallwood J, Jefferies E (2015) Automatic and controlled semantic retrieval: TMS reveals distinct contributions of posterior middle temporal gyrus and angular gyrus. J Neurosci 35:15230-15239. CrossRef Medline

Davidson PS, Anaki D, Ciaramelli E, Cohn M, Kim AS, Murphy KJ, Troyer AK, Moscovitch M, Levine B (2008) Does lateral parietal cortex support episodic memory? Evidence from focal lesion patients. Neuropsychologia 46:1743-1755. CrossRef Medline

Dede AJ, Wixted JT, Hopkins RO, Squire LR (2016) Autobiographical memory, future imagining, and the medial temporal lobe. Proc Natl Acad Sci U S A 113:13474-13479. CrossRef Medline

Devitt AL, Addis DR, Schacter DL 2017 Episodic and semantic content of memory and imagination: a multilevel analysis. Mem Cognit. In press. CrossRef Medline

Drowos DB, Berryhill M, André JM, Olson IR (2010) True memory, false memory, and subjective recollection deficits after focal parietal lobe lesions. Neuropsychology 24:465-475. CrossRef Medline

Gaesser B, Sacchetti DC, Addis DR, Schacter DL (2011) Characterizing agerelated changes in remembering the past and imagining the future. Psychol Aging 26:80-84. CrossRef Medline

Guerin SA, Robbins CA, Gilmore AW, Schacter DL (2012) Interactions between visual attention and episodic retrieval: Dissociable contributions of parietal regions during gist-based false recognition. Neuron 75:11221134. CrossRef Medline

Hassabis D, Kumaran D, Vann SD, Maguire EA (2007) Patients with hippocampal amnesia cannot imagine new experiences. Proc Natl Acad Sci U S A 104:1726-1731. CrossRef Medline

Hower KH, Wixted J, Berryhill ME, Olson IR (2014) Impaired perception of mnemonic oldness, but not mnemonic newness, after parietal lobe damage. Neuropsychologia 56:409-417. CrossRef Medline

Humphreys GF, Lambon Ralph MA (2015) Fusion and fission of cognitive functions in the human parietal cortex. Cereb Cortex 25:3547-3560. CrossRef Medline

Kim H (2010) Dissociating the roles of the default-mode, dorsal, and ventral networks in episodic memory retrieval. Neuroimage 50:1648-1657. CrossRef Medline

Kim H (2012) A dual-subsystem model of the brain's default network: Selfreferential processing, memory retrieval processes, and autobiographical memory retrieval. Neuroimage 61:966-977. CrossRef Medline

Kosslyn SM, Pascual-Leone A, Felician O, Camposano S, Keenan JP, L W, Thompson, Ganis G, Sukel KE, Alpert NM (1999) The role of area 17 in visual imagery: convergent evidence from PET and rTMS. Science 284: 167-170. CrossRef Medline

Kurczek J, Wechsler E, Ahuja S, Jensen U, Cohen NJ, Tranel D, Duff M (2015) Differential contributions of hippocampus and medial prefrontal cortex to self-projection and self-referential processing. Neuropsychologia 73:116-126. CrossRef Medline

Levine B, Svoboda E, Hay JF, Winocur G, Moscovitch M (2002) Aging and autobiographical memory: dissociating episodic from semantic retrieval. Psychol Aging 17:677-689. CrossRef Medline

Levy DA (2012) Towards an understanding of parietal mnemonic processes: some conceptual guideposts. Front Integr Neurosci 6:41. CrossRef Medline

Madore KP, Schacter DL (2016) Remembering the past and imagining the future: Selective effects of an episodic specificity induction on detail generation. Q J Exp Psychol (Hove) 69:285-298. CrossRef Medline

Madore KP, Gaesser B, Schacter DL (2014) Constructive episodic simula- 
tion: dissociable effects of a specificity induction on remembering, imagining, and describing in young and older adults. J Exp Psychol Learn Mem Cogn 40:609-622. CrossRef Medline

Morcom AM, Rugg MD (2012) Retrieval orientation and the control of recollection: an fMRI study. J Cogn Neurosci 24:2372-2384. CrossRef Medline

Moscovitch M, Cabeza R, Winocur G, Nadel L (2016) Episodic memory and beyond: the hippocampus and neocortex in transformation. Annu Rev Psychol 67:105-134. CrossRef Medline

Mueller S, Wang D, Fox MD, Yeo BT, Sepulcre J, Sabuncu MR, Shafee R, Lu J, Liu H (2013) Individual variability in functional connectivity architecture of the human brain. Neuron 77:586-595. Medline

Nilakantan AS, Bridge DJ, Gagnon EP, VanHaerents SA, Voss JL (2017) Stimulation of the posterior cortical-hippocampal network enhances precision of memory. Curr Biol 27:465-470. CrossRef Medline

Price AR, Peelle JE, Bonner MF, Grossman M, Hamilton RH (2016) Causal evidence for a mechanism of semantic integration in the angular gyrus as revealed by high-definition transcranial direct current stimulation. J Neurosci 36:3829-3838. CrossRef Medline

Race E, Keane MM, Verfaellie M (2011) Medial temporal lobe damage causes deficits in episodic memory and episodic future thinking not attributable to deficits in narrative construction. J Neurosci 31:1026210269. CrossRef Medline

Rorden C, Karnath HO, Bonilha L (2007) Improving lesion-symptom mapping. J Cogn Neurosci 19:1081-1088. CrossRef Medline

Rugg MD, Vilberg KL (2013) Brain networks underlying episodic memory retrieval. Curr Opin Neurobiol 23:255-260. CrossRef Medline

Rugg MD, Wilding EL (2000) Retrieval processing and episodic memory. Trends Cogn Sci 4:108-115. CrossRef Medline

Ryals AJ, Rogers LM, Gross EZ, Polnaszek KL, Voss JL (2016) Associative recognition memory awareness improved by theta-burst stimulation of frontopolar cortex. Cereb Cortex 26:1200-1210. CrossRef Medline

Schacter DL, Addis DR (2007) The cognitive neuroscience of constructive memory: remembering the past and imagining the future. Philos Trans $\mathrm{R}$ Soc Lond B Biol Sci 362:773-786. CrossRef Medline

Schacter DL, Addis DR, Buckner RL (2008) Episodic simulation of future events: concepts, data, and applications. Ann N Y Acad Sci 1124:39-60. CrossRef Medline

Schacter DL, Addis DR, Hassabis D, Martin VC, Spreng RN, Szpunar KK (2012) The future of memory: remembering, imagining, and the brain. Neuron 76:677-694. CrossRef Medline

Schacter DL, Addis DR, Szpunar KK (2017a) Escaping the past: contributions of the hippocampus to future thinking and imagination. In: The Hippocampus from cells to systems: structure, connectivity, and functional contributions to memory and flexible cognition. (Hannula DE, Duff MC, eds), pp 439-465. New York: Springer.

Schacter DL, Benoit RG, Szpunar KK (2017b) Episodic future thinking: mechanisms and functions. Curr Opin Behav Sci 17:41-50. CrossRef
Sestieri C, Capotosto P, Tosoni A, Luca Romani G, Corbetta M (2013) Interference with episodic memory retrieval following transcranial stimulation of the inferior but not the superior parietal lobule. Neuropsychologia 51:900906. CrossRef Medline

Sestieri C, Shulman GL, Corbetta M (2017) The contribution of the human posterior parietal cortex to episodic memory. Nat Rev Neurosci 18:183192. CrossRef Medline

Shimamura AP (2011) Episodic retrieval and the cortical binding of relational activity. Cogn Affect Behav Neurosci 11:277-291. CrossRef Medline

Simons JS, Peers PV, Mazuz YS, Berryhill ME, Olson IR (2010) Dissociation between memory accuracy and memory confidence following bilateral parietal lesions. Cereb Cortex 20:479-485. CrossRef Medline

Slotnick SD, Thakral PP (2011) Memory for motion and spatial location is mediated by contralateral and ipsilateral motion processing cortex. Neuroimage 55:794-800. CrossRef Medline

Squire LR, van der Horst AS, McDuff SG, Frascino JC, Hopkins RO, Mauldin KN (2010) Role of the hippocampus in remembering the past and imagining the future. Proc Natl Acad Sci U S A 107:19044-19048. CrossRef Medline

Stamenova V, Gao F, Black SE, Schwartz ML, Kovacevic N, Alexander MP, Levine B (2017) The effect of focal cortical frontal and posterior lesions on recollection and familiarity in recognition memory. Cortex 91:316326. CrossRef Medline

Stawarczyk D, D'Argembeau A (2015) Neural correlates of personal goal processing during episodic future thinking and mind-wandering: an ALE meta-analysis. Hum Brain Mapp 36:2928 -2947. CrossRef Medline

Thakral PP, Slotnick SD (2011) Disruption of MT impairs motion processing. Neurosci Lett 490:226-230. CrossRef Medline

Thakral PP, Benoit RG, Schacter DL (2017) Imagining the future: the core episodic simulation network dissociates as a function of time course and the amount of simulated information. Cortex 90:12-30. CrossRef Medline

Vilberg KL, Rugg MD (2008) Memory retrieval and the parietal cortex: a review of evidence from a dual-process perspective. Neuropsychologia 46:1787-1799]. CrossRef Medline

Wang JX, Rogers LM, Gross EZ, Ryals AJ, Dokucu ME, Brandstatt KL, Hermiller MS, Voss JL (2014) Targeted enhancement of corticalhippocampal brain networks and associative memory. Science 345:10541057. CrossRef Medline

Wende KC, Straube B, Stratmann M, Sommer J, Kircher T, Nagels A (2012) Neural correlates of continuous causal word generation. Neuroimage 62: 1399-1407. CrossRef Medline

Yazar Y, Bergström ZM, Simons JS (2014) Continuous theta burst stimulation of angular gyrus reduces subjective recollection. PLoS One 9:e110414. CrossRef Medline

Yazar Y, Bergström ZM, Simons JS (2017) Reduced multimodal integration of memory features following continuous theta burst stimulation of angular gyrus. Brain Stimul 10:624-629. CrossRef Medline 